\title{
Dynamic loading of a water jet propulsion drive of amphibious vehicles
}

\author{
Sergey Abdulov ${ }^{1}$, Alexander Taratorkin ${ }^{2}$, and Pavel Nenashev ${ }^{1, *}$ \\ ${ }^{1}$ Federal State Budgetary Educational Institution of Higher Education "Kurgan state university", \\ 63S4, Sovetskaya street, Kurgan, 640020, Russia \\ ${ }^{2}$ Institute of Engineering Science, Ural Branch of the Russian Academy of Sciences, 34, \\ Komsomolskaya street, Ekaterinburg, 620049, Russia
}

\begin{abstract}
The analysis results of dynamic loading of the amphibious vehicle' water jet drive are presented, the hypothesis about the durability limitation of the drive due to the onset of resonance forced and parametric oscillations is advanced. Solutions of that problem are justified.
\end{abstract}

In designs of transmissions of all-wheel-drive wheeled vehicle and tractors, amphibious vehicles and others the divided mechanical drives with spatially arranged drivelines (cardan drives) are used. Field experience and results of the experimental efforts of vehicles' prototypes with such drives indicate limited durability of elements while safety factors are sufficiently. Limited durability of drives' elements become apparent in damages of shafts' splined connections, angle gearboxes, water jets, impellers, etc. The analysis of methods of design calculations demonstrates that the well-known techniques do not pay due attention to the estimation of specifics of drives dynamic loading.

This work considers theoretic and experimental research of drives dynamic loading as well as justification of ways of its decrease.

The objective is achieved by dynamical and mathematical models of systems, simulation modeling of dynamics, oscillating processes analysis with variation of parameters that provide system dynamic stability. Research is performed using the amphibious vehicle, its mobility on the water is provided by water jets use. This solution provides also maneuverability - ability to control of movement direction including reverse movement. Alongside with functional requirements for water jet drives of the amphibious vehicles the special reliability requirements are imposed since failure of the one of elements leads to vehicle mobility disturbance on the water.

Kinematic scheme of the drive is given in Figure 1. Water jets drive with quadruple threaded screw of impellers, that placed under waterline, is fulfilled by drivelines from Left (L) and Right (R) of transmission output shafts with rotational speed 1.467 times more than rotational speed of engine $\dot{\varphi}_{E}$. Due to the layout, the transmission axis is displaced relative vehicle centerline. In this regard, the installation angles of drivelines on each side are different (Table 1).

* Corresponding author: pasteer@mail.ru 


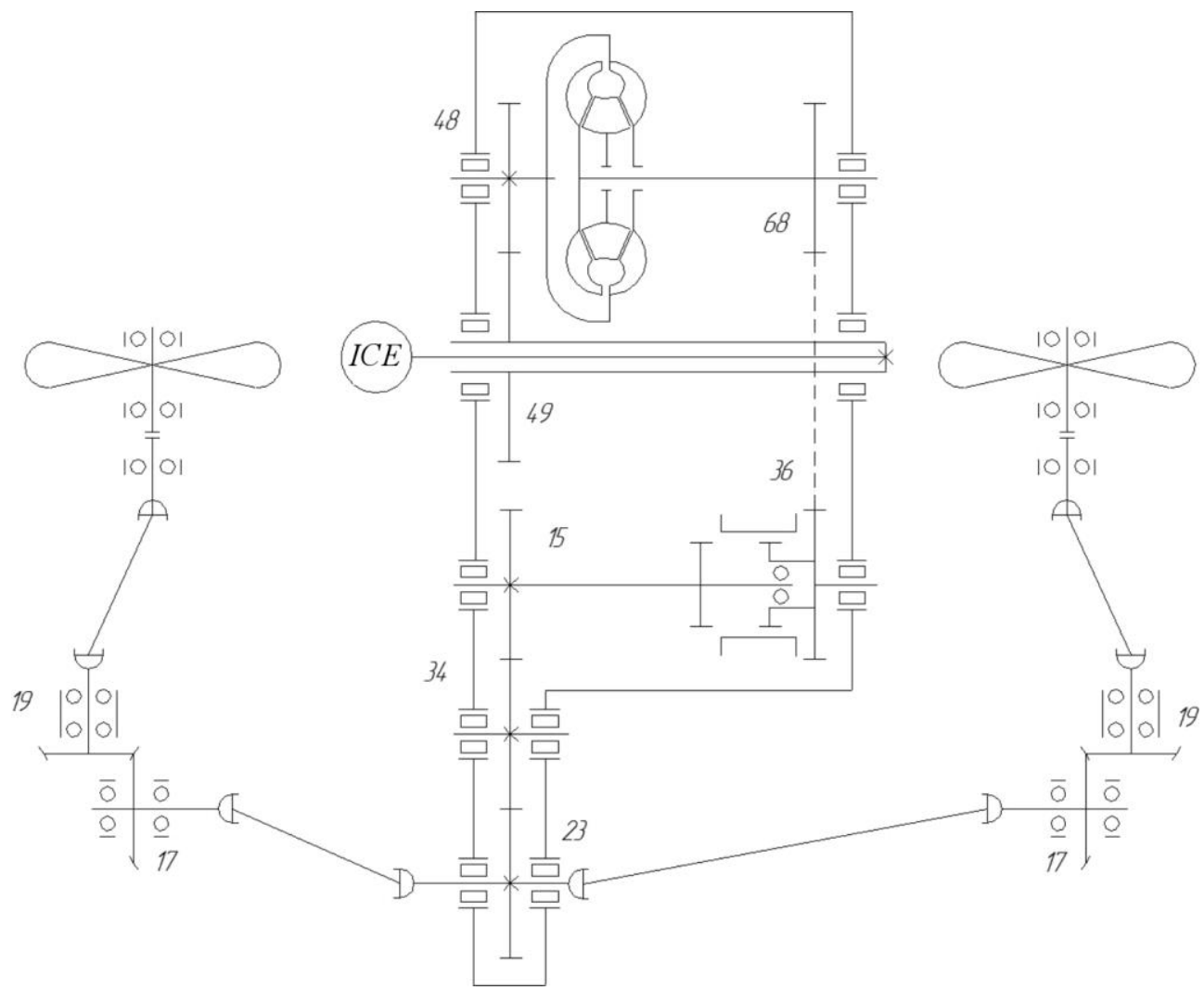

Fig. 1. Kinematic scheme of the drive.

Table 1. Values of angular coordinates that determine the spatial arrangement of drivelines

\begin{tabular}{|l|c|c|c|c|}
\hline & \multicolumn{2}{|c|}{ Left side } & \multicolumn{2}{c|}{ Right side } \\
\cline { 2 - 5 } & shaft 1 & shaft 2 & shaft 1 & shaft 2 \\
\hline $\begin{array}{l}\text { Angle } \gamma_{1} \\
\text { (horizontal plane) }\end{array}$ & $3^{\circ} 42^{\prime}$ & $16^{\circ} 43^{\prime}$ & $2^{\circ} 28^{\prime}$ & $16^{\circ} 43^{\prime}$ \\
\hline $\begin{array}{l}\text { Angle } \gamma_{2} \\
\text { (vertical plane) }\end{array}$ & $7^{\circ} 29^{\prime}$ & $10^{\circ} 27^{\prime}$ & $4^{\circ} 15^{\prime}$ & $10^{\circ} 27^{\prime}$ \\
\hline
\end{tabular}

The gear ratio of bevel gear of angle gearbox is $u_{a v}=0.8947$. When torque converter is interlocked the overall gear ratio of water jet drive from engine shaft to water jets impellers is $u_{d r}=0.711$.

Dynamical model reflects interconnection of all elements, their input, output characteristics, and consists of mathematical models of functional systems and units of transmission of vehicle including water jets drives. Such model complicates calculation procedure significantly, however, capabilities of modern computers and software allow to develop computation program that is capable without structural decomposition [1] of tested system to solve differential equation systems. 
Application of that mathematical model of dynamical processes in "engine transmission - transport vehicle" system is realized in sequential study of the specific, most indicative transient running regimes of transmission with the help of computers by specifically developed program from MATLAB package in Simulink Driveline application [2]. Block diagram of software implementation is given in Figure 2.

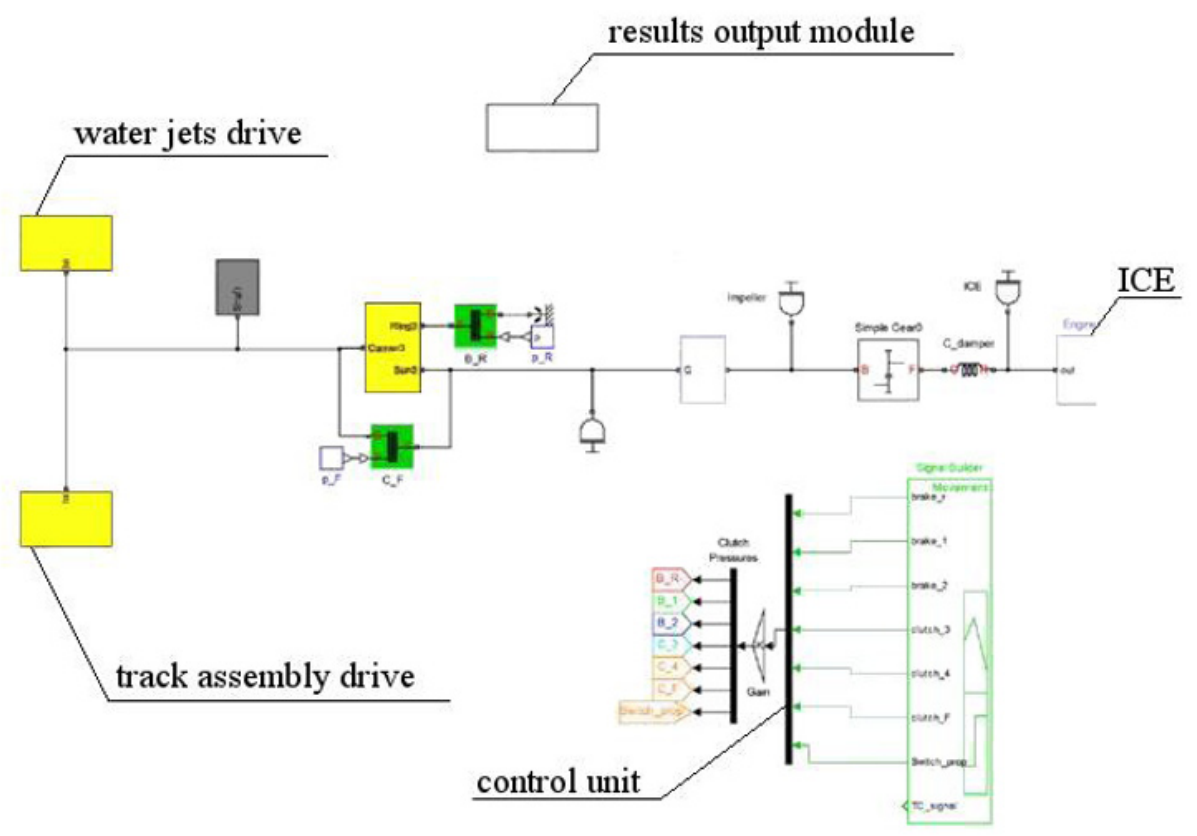

Fig. 2. Block diagram of the water jet's drive dynamic loading program(MATLAB package, Simulink Driveline application)

Developed mathematical model allows to:

- $\quad$ determine the characteristics of dynamic loading of water jet drives elements used for their loading spectra development and durability evaluation of relevant elements;

- determine the slipping characteristics of friction elements used for evaluation of their operability and durability;

- determine the processes of time variations of rotational speed and accelerations of the separate elements of transmission used for the evaluation of quality of transient running regimes.

The above listed characteristics are determined for following transient process: water jets acceleration ashore and afloat; vehicle's water entering/leaving when simultaneous operation of track assembly and water jets; reverse gear switching, etc., as well as steadystate movement regimes at constant speed. As an example of computation results, the time function of dynamic torque on the drive shaft of the water jet when switching on/off of the reverse gear and in steady-state regime is given in Figure 3. 

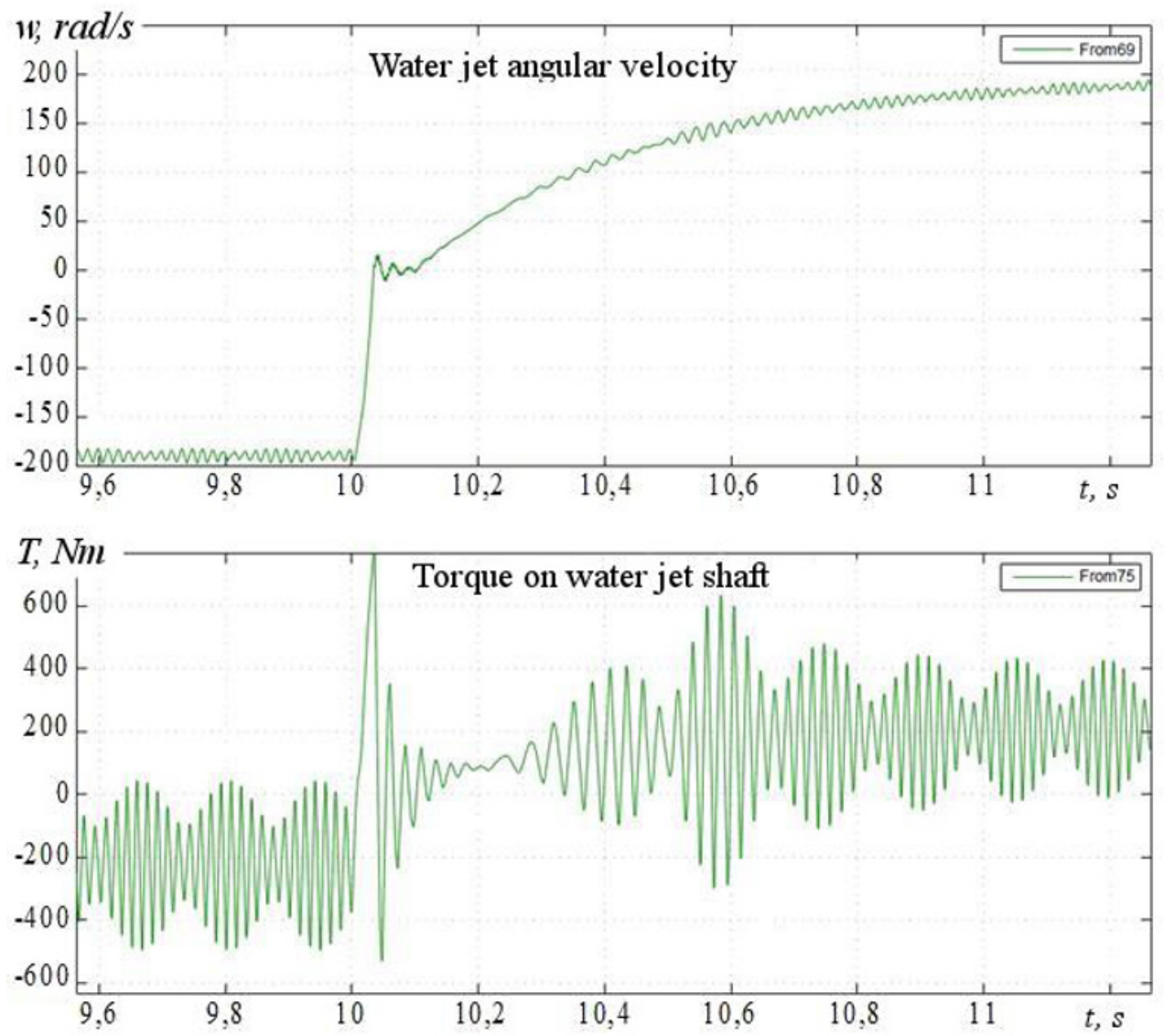

Fig. 3. Time function of dynamic torque on the drive shaft of the water jet when switching on/off of the reverse gear and in steady-state regime

Represented results of simulation demonstrate the possibility of emergence of specific forms of oscillating processes in the system (runouts, parametric oscillations) in steadystate regime. In this case, the oscillation frequency corresponds shaft's double rotational speed. In transient processes of the reverse gear switching on/off the dynamic factor increases to $1.9 \ldots 3.0$.

During the simulation a number of assumptions was made, correctness of which, as well as identification of system's separate parameters were determined during experimental studies in the process of the amphibious vehicle movement. During experimental studies the rotational speed of engine shaft and dynamic torque on connecting shafts of both hull sides were recorded. Analysis of the results of experimental studies of dynamic loading at transient processes showed that dynamic factor in all regimes is less than $1.1 \ldots 1.2$ except the reverse gear switching-on regime when dynamic factor reaches value 1.9...2.5 (Figure 4). 


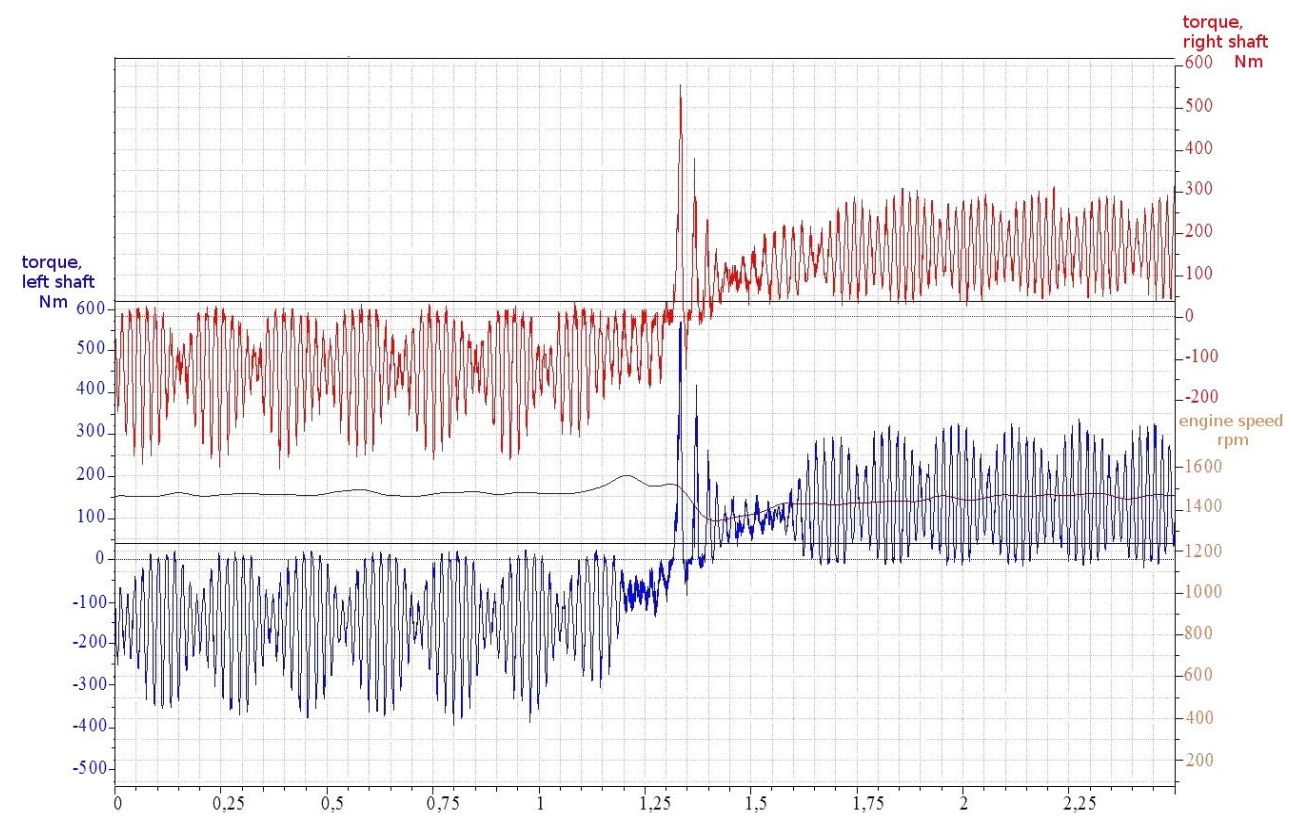

Fig. 4. Fragment of the oscillogram of dynamic torque changing on connecting shafts when water jet operates in the reverse gear switching-on regime and steady-state regime.

In steady-state regimes, the engine shaft rotational speed was changed discretely in the range from minimum stable 800 to 2370 with 200-rpm intervals. Fragment of the oscillogram of dynamic torque changing and the spectral density are given in Figure 5.
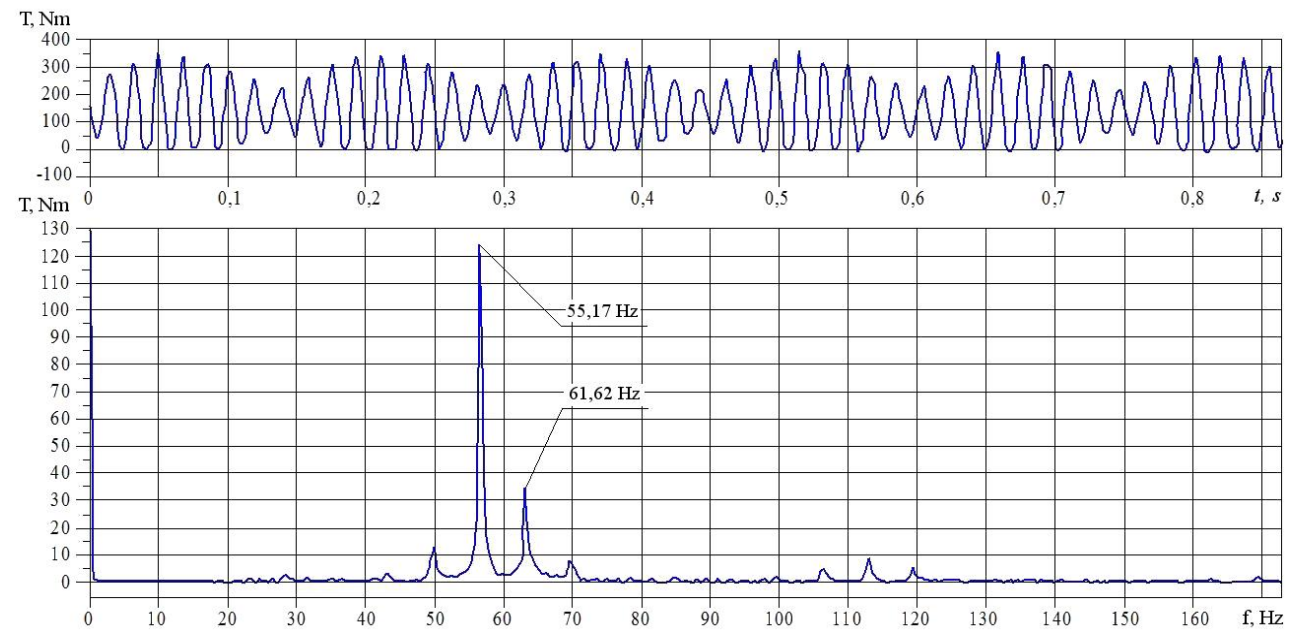

Fig. 5. Fragment of the oscillogram of dynamic torque changing and the spectral density of the process when water jet operates in steady-state regime

Experimental data analysis indicates that oscillating process has "runouts nature" that caused by summing of periodic components of the torque with near frequency. This is defined by gear ratio value of angle gearbox (19/17); therefore frequencies of periodic components of two drive shafts of the one hull side in all engine speed regimes are distinct from each other by $11.7 \%$. The fact that torque sensor mounted on the one shaft records 
periodic component of the torque which is formed by other shaft indicates that in mathematical model you need consider mutual spatial arrangement of joints of drivelines (cardan) of water jets drives.

Amplitude-rotational characteristic of the dynamic torque is given in Figure 6.

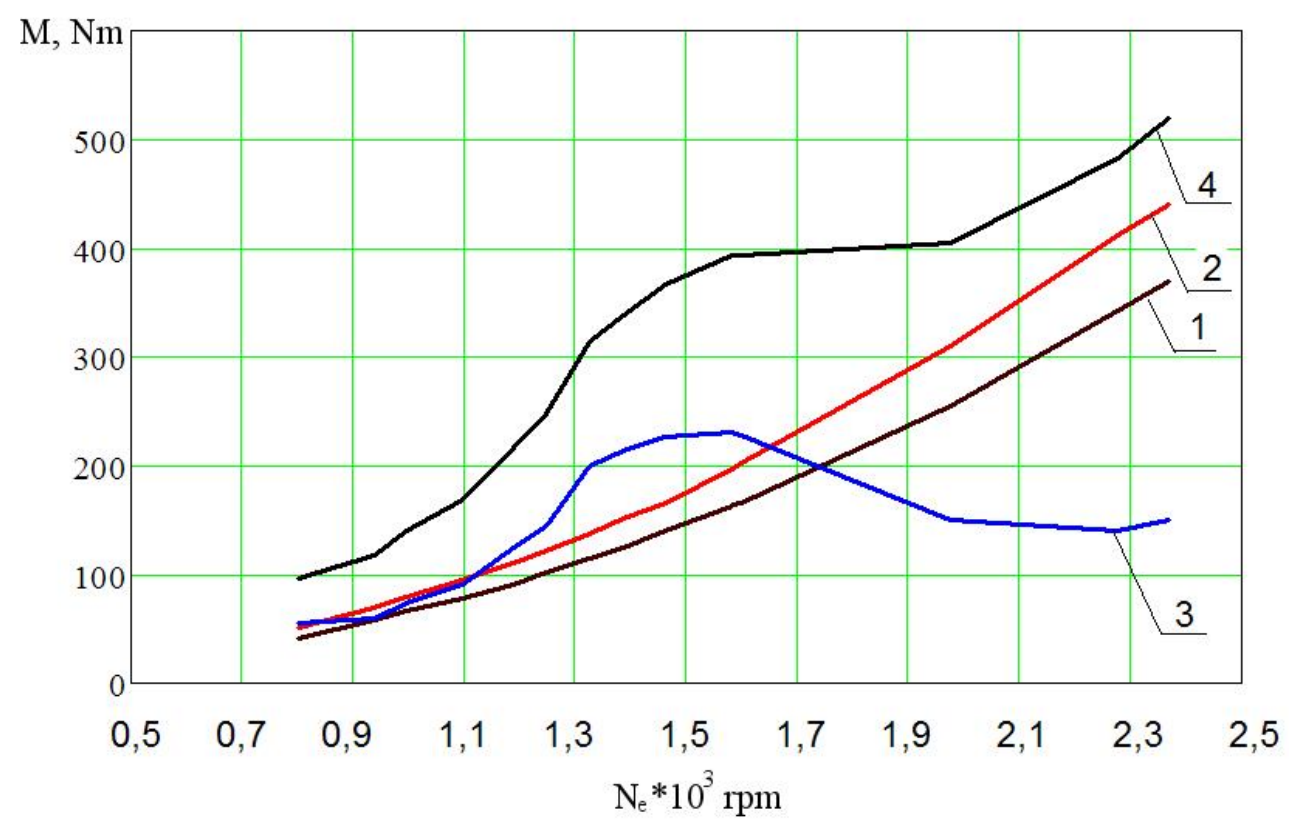

Fig. 6. Amplitude-rotational characteristic of dynamic torque of water jet drive

Above listed data shows that the average value of torque on drive shafts when rotational speed changing from 800 to $2370 \mathrm{rpm}$ increases according to quadratic dependence for left shaft (Curve 1 in Figure 7) from 41.4 to $370 \mathrm{Nm}$, and for right shaft (Curve 2 in Figure 7) from 50.9 to $440 \mathrm{Nm}$, i.e. at full engine speed the value of average torque on the right shaft is 1.19 times more than on the left one. At that, oscillating process, which forms dynamic torque, comes with frequency equal to doubled cardan one. Maximum values of dynamic component are observed at engine shaft rotational speed $1300 \ldots 1500 \mathrm{rpm}$ and are $230 \mathrm{Nm}$ (Curve 3 in Figure 6). Maximum value of the dynamic torque that represents the sum of the average torque and variable component's amplitude is changed according to Curve 4. Comparison of results of numerical simulation and experimental studies coincides with sufficient accuracy at confidence probability minimum $95 \%$. Nature of dynamic torque changing, as well as analysis of its amplitude and frequency characteristic in all speed range of engine indicates about excitation of parametric oscillations in the system that limit the elements durability.

Parametric oscillations and resonances are dangerous phenomena, as they take place in wide range of disturbing frequency with exponentially increasing amplitudes of the dynamic torque. In the case of the parametric oscillations, the drive design is subjected to dangerous cyclic loading that can lead to fatigue breakdown of the drive elements. Therefore, the main task of the design dynamic analysis in which parametric resonances are excited is determination of the boundaries of the areas of dynamic instability in order to take measures for parametric resonance elimination when reworking.

In the mechanical system under consideration, the parameters formed by drivelines with asynchronous joints are periodically changed. The oscillation amplitude of dynamic torque in the drive is limited in this case and significant resistance moment when the vehicle is on the water does not allow to expand the backlash in the bevel gear of angle gearbox. 
Therefore when analyzing the parametric oscillations excited by drivelines the drive is considered as linear system. Coupling transmission and angle gearboxes of the water jet drives is performed by drivelines with asynchronous joints. In the vertical plane the left shaft axis is located relative to the horizontal at angle $\gamma_{L}=7^{\circ} 29^{\prime}$ ' , and the right one $\gamma_{R}=4^{\circ} 15^{\prime}$. Gear ratio of the asynchronous joints is variable due to periodic changing in angular velocity $\dot{\varphi} 1$ of the driven joint fork from maximum $\dot{\varphi} 1 / \cos \gamma$ to minimum value $\dot{\varphi} 1 \cdot \cos \gamma$ twice per one rotation of joints relative to the constant speed of the driving joint fork $\dot{\varphi} 1$. Hence, driven joint fork and input shaft of angle gearbox rotate at speed $\dot{\varphi} 2=$ $\dot{\varphi} 1+\Delta \dot{\varphi} 1 \cdot \cos (2 \dot{\varphi} 1 \cdot \mathrm{t})$, where $\Delta \varphi=\left(\frac{\varphi 1}{\cos \gamma}-\varphi 1 \cdot \cos \gamma\right)=\varphi 1 \cdot \sin ^{2} \gamma / \cos \gamma$. Without considering the influence of oscillations on movement of the vehicle inertia mass the differential equation of the system's relative movement is transformed to the form $J_{R} \varphi+$ $c\left(1+\frac{\Delta \varphi_{1}}{\varphi_{1}} \sin \dot{\varphi} \cdot t\right) \varphi=M_{E}-M_{R}$. This equation differs from the common one in that the elastic moment contains periodically changed parameter, i.e. this equation corresponds to the form of the Mathieu differential equation of parametric oscillations [3].

To analyze the stability of the parametric oscillations the Mathieu equation is transformed to the form of parameters of the Ince-Strutt diagram $+\omega_{0}^{2}[\alpha+2 h \cos (2 \tau)] \varphi=$ 0 , where $\alpha$ - abscissa, $h$ - diagram ordinate. Abscissa is determined by ratio of frequencies, natural and exciting $p, \alpha=\left(2 \omega_{0} / p\right)^{2}, \omega_{0}^{2}=c / J_{R}, p=2 \dot{\varphi}, 2 \tau=2 \dot{\varphi}$. Diagram ordinate $h=\alpha \mu$, where $\mu$ - parameter of the modulation depth $\mu=\Delta \varphi / \varphi_{a v}, \varphi_{a v}=\left(M_{E}-\right.$ $\left.M_{R}\right) / c$. Ince-Strutt diagram is given in Figure 7, stability region is shaded.

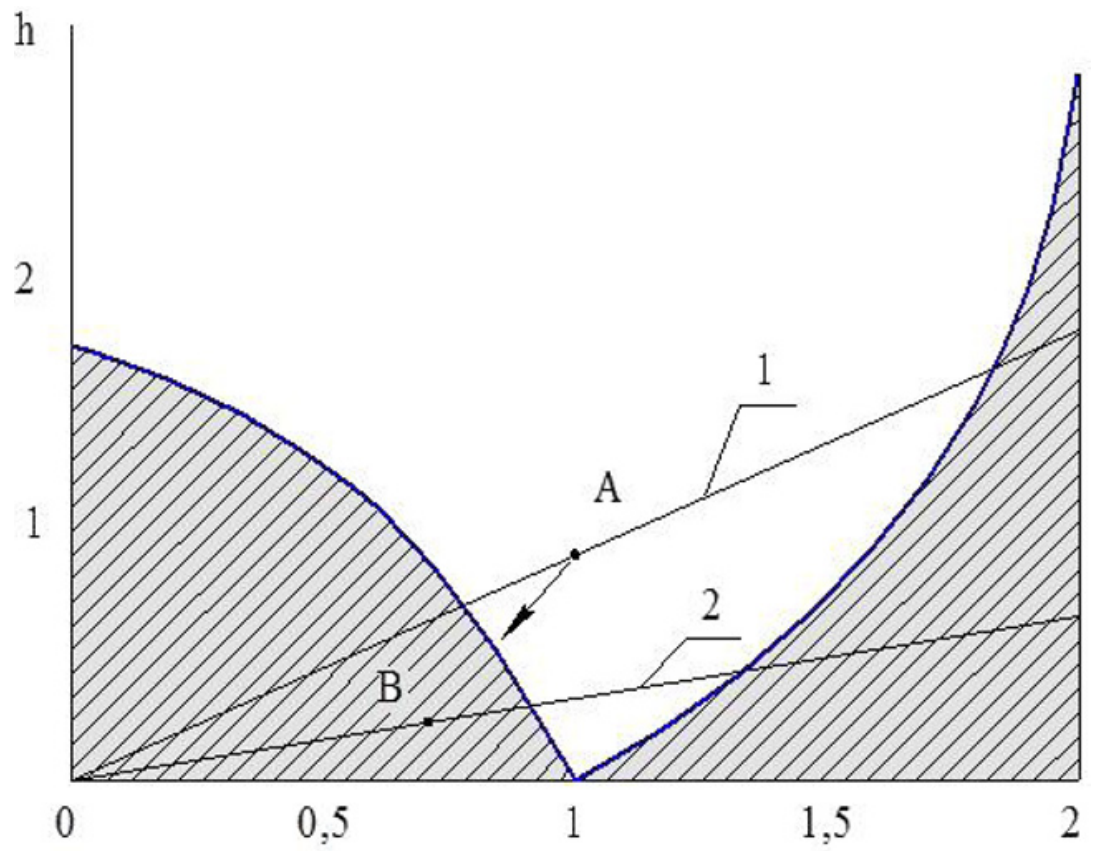

Fig. 7. Ince-Strutt diagram.

Analysis of the stability of parametric oscillations is made according to function $h=\alpha \mu$ of the considered system. In the diagram Line 1 corresponds to the parameters of the left drive $\left(\gamma_{L}=7^{\circ} 29^{\prime}, c=7780 \mathrm{Nm} / \mathrm{rad}\right.$. Line $h=\alpha \mu$ crosses alternating regions of stability and instability. Parametric resonances are possible in the instability region. Graph 1 of the diagram shows that Line $h=\alpha \mu$ crosses a wide region of instability. This means higher probability of dynamic stability loss, practically at any technically possible value of 
disturbing frequencies. Reduced resonance $(\alpha=1)$ is the main and most dangerous. In this case the ratio of disturbing frequencies and nature one is $p=2 \omega_{0}$.

The above refers to the conditions where energy dissipation is absent. This is the case when water jets drives operate on land - before entering and leaving the water. When afloat the water jets operation is accompanied with significant energy dissipation. In this case the amplitude of parametric oscillations is most significant in regime of the first main resonance $\left(\alpha=1, p=2 \omega_{0}\right)$ the most dangerous and low sensitive to the action of the dissipative forces. The critical value of frequency is $p=2 \omega_{0} \sqrt{1-\frac{1}{2}\left(\frac{\Delta}{\pi}\right)^{2}} \pm \sqrt{\mu^{2}}-\left(\frac{\Delta}{\pi}\right)^{2}+$ $\frac{1}{4}\left(\frac{\Delta}{\pi}\right)^{2}$, where $\Delta$ - damping decrement, $\Delta=\frac{2 \pi \varepsilon}{\varphi_{0}}, \varepsilon$ - damping factor. System stability can be enhanced by the introduction of oscillation absorber, i.e. additional compliance of one of the drive elements, such as gear wheel of transmission. Absorber adjustment allows to tune away the resonance, excluding getting into the dynamic instability region at certain value of the modulation depth.

Another effective way to eliminate parametric oscillations is to decrease the modulation parameter. This is achieved by limiting of installation angle of drivelines with asynchronous joints. If the layout does not allow that, then it is necessary to introduce constant velocity joints (CVJ) for which the modulation parameter of the angular velocity is an order of magnitude less than for asynchronous ones. In Figure 8 Line 2 corresponds to left drive parameters with constant velocity joint. It follows from an analysis of arrangement of Line 2 on Ince-Strutt diagram that the drive with CVJs practically eliminates the probability of excitation of the parametric oscillations.

\section{Conclusions}

Reduction of dynamic loading generated by parametric resonances can be achieved by the introduction of the absorber drive as well as constant velocity joints that predetermines the durability increasing of the drive elements.

Damping of the oscillation amplitudes of the dynamic torque acting in the water jet drive that contains spatially arranged drivelines, as well as the increasing of the durability of its elements is also achieved by mutual arrangement of the driveline elements during assembling.

\section{References}

1. V. Algin, Systematization and calculation of mobile vehicle as multimass system. Dynamics of machine unit. Mechanics of vehicles, mechanisms and materials. International scientific and technical journal. Minsk. Belarus, v. 2(23),pp. 5-18 (2013)

2. I. Chernykh, Simulation of electrical devices with MATLAB, SimPowerSystems and Simulink. 1-st edition, (2007), ISBN 978-5-388-00020-0.

3. Y. Panovko, Introduction to the theory of mechanical vibrations: study guide, revised 2-nd edition, M. Nauka, Main office of physical and mathematical literature, pp. 272 (1980) 